\title{
S-process in hot AGB stars: A complex interplay between diffusive mixing and nuclear burning
}

\author{
S. Goriely and L. Siess \\ Institut d'Astronomie et d'Astrophysique, Université Libre de Bruxelles, CP 226, 1050 Brussels, Belgium \\ Received 18 February 2004 / Accepted 25 May 2004

\begin{abstract}
We present new qualitative results of the s-process nucleosynthesis in hot AGB stars resulting from diffusive convective overshooting. In this approach, protons from the convective envelope are injected into the adjacent ${ }^{12} \mathrm{C}$-rich layers, but depending on the thermodynamic conditions in the mixing zone, the nuclear proton lifetime can be comparable with (or even smaller than) the diffusion timescale. In such a case, the nucleosynthesis and diffusion equations must be coupled. Our simulations indicate that during this diffusive burning phase, the ${ }^{14} \mathrm{~N}$-rich and ${ }^{13} \mathrm{C}$-rich layers overlap with drastic impact on the s-process distribution. Our study emphasizes the sensitivity of the s-process nucleosynthesis on the temperature of the partial mixing zone. We show that in this framework, a deep hot third dredge-up tends to inhibit the production of s-elements. We also provide a first qualitative estimate of the metallicity and mass dependence of the s-process efficiency in AGB stars and propose that this complex interplay could account for the large variety of the observed s-process efficiencies.
\end{abstract}

Key words. nucleosynthesis - s-process - AGB stars

\section{Introduction}

The global mechanism responsible for the nucleosynthesis of the s-elements is well understood: successive neutron captures on heavy nuclei, starting mostly from the Fe-group elements, are followed by beta decays, with a neutron capture time scale exceeding the half-lives of most of the unstable nuclei. In AGB stars, the neutrons required for this process are mainly produced by $\alpha$-captures on ${ }^{13} \mathrm{C}$ in a thin radiative layer below the convective envelope. Although parametric studies, by artificially incorporating suitable amounts of ${ }^{13} \mathrm{C}$ into the system (Gallino et al. 1998; Busso et al. 2001) or by injecting protons into the C-rich layers at the time of the third dredge-up (Goriely \& Mowlavi 2000) are able to reproduce a large set of observed heavy elements abundances, the very origin of this so-called " ${ }^{13} \mathrm{C}$ pocket" remains the central problem of realistic s-process models. It has long been suggested (Iben \& Renzini 1982), that if protons from the envelope can mix partially with the carbon-rich layers left behind the pulse-driven convective shell, then ${ }^{13} \mathrm{C}$ can be synthesized through the chain of reactions ${ }^{12} \mathrm{C}(\mathrm{p}, \gamma){ }^{13} \mathrm{~N}\left(\beta^{+}\right){ }^{13} \mathrm{C}$. However, the physical mechanism responsible for injecting protons into the ${ }^{12} \mathrm{C}$-rich layers is still largely unknown.

The most recent attempts to identify the physical cause of this extra mixing refer to diffusive convective overshooting (Herwig et al. 1997; Goriely \& Mowlavi 2000), rotationally induced mixing (Langer et al. 1999; Herwig et al. 2003;

Send offprint requests to: S. Goriely, e-mail: sgoriely@astro.ulb.ac.be
Siess et al. 2004) or gravity waves (Denissenkov \& Tout 2003). The best studied mechanism so far is the overshoot model, which is able to produce s-process abundance patterns consistent with many observations (Lugaro et al. 2003; Van Eck et al. 2003). However, some major problems still remain with this approach. In particular, the size of the partial mixing zone induced by convective overshooting - about $10^{-5} M_{\odot}$ - is one order of magnitude too small to explain the observed barium surface overabundances of up to 100 in AGB stars (Goriely \& Mowlavi 2000). In addition, although the predictions of the overshoot scenario agree remarkably well with recent observations of low-metallicity Pb-stars (Van Eck et al. 2001), they fail to reproduce another class of s-process-rich low-metallicity stars that are depleted in $\mathrm{Pb}$ (e.g., Aoki et al. 2002; Van Eck et al. 2003). Therefore, further studies of the s-process in AGB stars are required.

In most stellar evolution calculations, the nuclear and diffusion processes are decoupled since mixing is generally much faster than nuclear burning. However when the nuclear and diffusion timescales become comparable, this simplified hypothesis breaks down and a fully coupled scheme must be used. The present paper aims at re-visiting the s-process nucleosynthesis induced convective overshooting in stars presenting 3DUP characteristics that require the coupling of nuclear and diffusion processes. The plan of our paper is as follows: in the next section we describe the input physics of the stellar evolution and post-processing codes. Section 2 reviews the s-process nucleosynthesis in the overshoot model, and in Sect. 3 we analyze the effects of coupling diffusion and nuclear 
burning on the resulting s-process efficiency for different values of the overshoot depth in one interpulse phase of a $3 M_{\odot} Z=$ 0.0001 AGB model star. In Sect. 4, we estimate qualitatively the dependence of the s-process efficiency on the metallicity and mass of AGB stars.

\section{The s-process in the overshoot model}

The key concern for the s-process is the partial mixing of protons into the $\mathrm{C}$-rich layers. This process is known to take place in regions where the proton to carbon abundances ratios range between $0.01 \lesssim Y_{\mathrm{p}} / Y_{{ }^{12} \mathrm{C}} \lesssim 1$ (where $Y_{i}$ is the molar mass fraction of isotope $i$ ). In this regime, ${ }^{12} \mathrm{C}$ is burned into ${ }^{13} \mathrm{C}$ by proton captures, but the amount of protons is too small to complete the $\mathrm{CN}$ cycle through ${ }^{13} \mathrm{C}(\mathrm{p}, \gamma){ }^{14} \mathrm{~N}$, so that the abundance of the neutron poison ${ }^{14} \mathrm{~N}$ remains lower than ${ }^{13} \mathrm{C}$. After the formation of this ${ }^{13} \mathrm{C}$-rich ${ }^{14} \mathrm{~N}$-poor layers, later during the interpulse period the temperature increase to $T_{8} \simeq 0.9$ (where $T_{8}$ is the temperature expressed in $10^{8} \mathrm{~K}$ ) ignites the neutron source ${ }^{13} \mathrm{C}(\alpha, \mathrm{n}){ }^{16} \mathrm{O}$. Contrary to the production of the primary ${ }^{13} \mathrm{C}$ and ${ }^{14} \mathrm{~N}$, the s-process is remarkably sensitive to the stellar metallicity $Z$ for the simple reason that the seed (mainly iron) abundance is proportional to $Z$, while the primary ${ }^{13} \mathrm{C}$ abundance is metallicity-independent. As a result, the neutron-to-seed abundance ratio is inversely proportional to $Z$, and the s-process efficiency increases with decreasing metallicities. Below a given metallicity (typically, $Z \lesssim 0.001$ ), the instantaneous mixing of protons predicts that most of the seed nuclei are transformed into $\mathrm{Pb}$ and $\mathrm{Bi}$, the heaviest elements possibly produced by the s-process. This result led to the prediction of the existence of metal-poor s-enriched $\mathrm{Pb}$-stars which was later confirmed (Van Eck et al. 2001).

The partial mixing of protons into the C-rich layers can be generated assuming some overshoot mixing beyond the convective boundary. In the time-dependent diffusive approach of the overshoot (Herwig et al. 1997), the diffusion coefficient below the convective envelope is given by

$D_{\text {over }}\left(r<r^{\text {env, bot }}\right)=D_{\text {conv }}^{\text {env, bot }} \times \mathrm{e}^{\frac{\left(r^{\text {env, bot }-r}\right)}{f_{\text {over }} \times H_{\mathrm{p}}^{\text {env,bot }}}}$

where $r^{\text {env,bot }}$ and $H_{\mathrm{p}}^{\text {env,bot }}$ are the radius and pressure scale height at the bottom of the convective envelope, and $f_{\text {over }}$ a free parameter characterizing the extent of the diffusive region. The success of the overshoot model comes from the exponentially decreasing character of the diffusion coefficient (Eq. (1)) which enables the formation of a $Y_{\mathrm{p}} / Y_{{ }^{12} \mathrm{C}} \sim 0.1$ pocket of relevance to the s-process nucleosynthesis at the radial location corresponding to $D_{\text {over }} \sim 10^{4}-10^{6} \mathrm{~s} / \mathrm{cm}^{2}$. Above this region up to the convective envelope where $D_{\text {over }}=D_{\text {conv }} \simeq 10^{14} \mathrm{~s} / \mathrm{cm}^{2}$, diffusion is efficient and $Y_{\mathrm{p}} / Y_{{ }^{12} \mathrm{C}} \gtrsim 1$ leading to a complete $\mathrm{CN}$ cycle and the dominant production of ${ }^{14} \mathrm{~N}$. As long as the diffusion is not affected by nuclear burning, the choice adopted for the free parameter $f_{\text {over }}$ does not affect the s-process abundance distribution, but only the extent of the s-enriched region, i.e the global s-process overproduction.

However, in low-metallicity and/or massive AGB stars, the region in which the partial mixing occurs can reach relatively high temperatures at the time of the 3DUP (Herwig 2004). In this case, the nuclear proton lifetime in this "hot" C-rich environment can be comparable with (or even smaller than) the diffusion timescale. For a typical ${ }^{12} \mathrm{C}$ mass fraction of 0.2 in a layer of density $\rho=1 \mathrm{~g} / \mathrm{cm}^{3}$, the proton lifetime against the ${ }^{12} \mathrm{C}(\mathrm{p}, \gamma){ }^{13} \mathrm{~N}$ reaction equals $\tau_{\mathrm{p}}=2193,19,1 \mathrm{yr}$ at a temperature of $T_{8}=0.4,0.6,0.8$, respectively. This is to be compared with the diffusion timescale between two adjacent layers distant typically of $\Delta r=10^{7} \mathrm{~cm}$, i.e $\tau_{\text {dif }} \simeq \Delta r^{2} / D_{\text {over }}=$ $3 \times 10^{-3}, 3,3000 \mathrm{yr}$ for $D_{\text {over }}=10^{9}, 10^{6}$ and $10^{3} \mathrm{~s} / \mathrm{cm}^{2}$, respectively.

To study the complex interplay between the diffusive and nuclear processes, a fully coupled approach is followed by solving the system of equations

$\frac{\partial Y_{i}}{\partial t}=$ nuclear terms $+\frac{\partial}{\partial m_{r}}\left[\left(4 \pi r^{2} \rho\right)^{2} D \frac{\partial Y_{i}}{\partial m_{r}}\right]$,

where $D=D_{\text {conv }}+D_{\text {over }}$ is the sum of the Eulerian convective and overshoot diffusion coefficients, respectively. This method (see Siess et al. 2004 for details) is presently used to perform post-processing s-process calculations as detailed in the next section.

\section{The s-process in the $\mathbf{3} M_{\odot} Z=0.0001$ model star}

The overshoot and resulting proton mixing in the $\mathrm{C}$-rich layers during the 16th interpulse-pulse sequence of a $3 M_{\odot}$ $Z=0.0001$ model star is now studied. This model star is selected because the base of its envelope is characterized by a temperature of the order of $T_{8} \simeq 0.4$ at the time of the 3DUP for which the diffusion and nuclear processes start to compete. In the reference model, no overshoot is implemented (this inconsistency will be further discussed in Sect. 4).

In Figs. 1 and 2 , we show the ${ }^{13} \mathrm{C}$ and ${ }^{14} \mathrm{~N}$ abundance profiles resulting from the diffusive overshoot with $f_{\text {over }}=0.01$ and $f_{\text {over }}=0.06$ at the end of the 3DUP phase which lasts about $200 \mathrm{yr}$. In the case of $f_{\text {over }}=0.01$, protons are mixed over a relatively small mass range of $\Delta M \simeq 10^{-5} M_{\odot}$ and during the diffusion process the temperature increases from $T_{8} \simeq 0.4$ to $T_{8} \simeq 0.54$. Comparisons with the instantaneous diffusion case, i.e excluding H-burning during the diffusion process (dotted lines), show that the diffusion process remains faster than the nuclear burning and consequently the instantaneous diffusion approximation remains valid. The ${ }^{13} \mathrm{C}$ pocket is seen to extend over some $10^{-6} M_{\odot}$, but the region of interest for the s-process is restricted to a mass range of about $2-3 \times 10^{-7} M_{\odot}$ where the ${ }^{13} \mathrm{C}$ mass fraction is larger than the ${ }^{14} \mathrm{~N}$ mass fraction and still significant (i.e. larger than about $10^{-3}$ ). At the end of the 3DUP phase, these ${ }^{13} \mathrm{C}$ and ${ }^{14} \mathrm{~N}$ profiles will lead to an efficient s-process characteristic of the low-metallicity $\mathrm{Pb}$-stars as seen in Fig. 3.

In Fig. 2, the ${ }^{13} \mathrm{C}$ and ${ }^{14} \mathrm{~N}$ abundance profiles result from a stronger overshooting with $f_{\text {over }}=0.06$ which diffuses protons down to deeper and hotter layers. The overshoot mass region extends over $\Delta M \simeq 10^{-4} M_{\odot}$ and the temperatures range between $T_{8} \simeq 0.5$ and $T_{8} \simeq 0.7$. In such a hot environment, the nuclear H-burning competes with the diffusion process, so that ${ }^{13} \mathrm{C}$ and ${ }^{14} \mathrm{~N}$ are produced while diffusion is still active. 


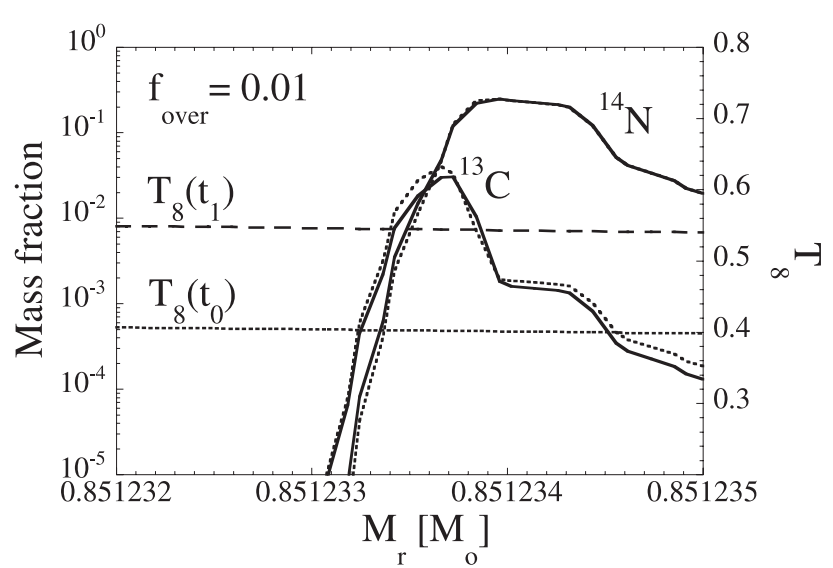

Fig. 1. ${ }^{13} \mathrm{C}$ and ${ }^{14} \mathrm{~N}$ profiles (solid lines) resulting from the diffusive overshoot with $f_{\text {over }}=0.01$ at the end of the 3DUP phase. The dotted lines correspond to the ${ }^{13} \mathrm{C}$ and ${ }^{14} \mathrm{~N}$ profiles that would have been obtained without considering the coupling between the nuclear and diffusion processes. Also given are the temperatures in the corresponding mass region at the beginning, $T_{8}\left(t_{0}\right)$, and at the end, $T_{8}\left(t_{1}\right)$, of the 3DUP phase.

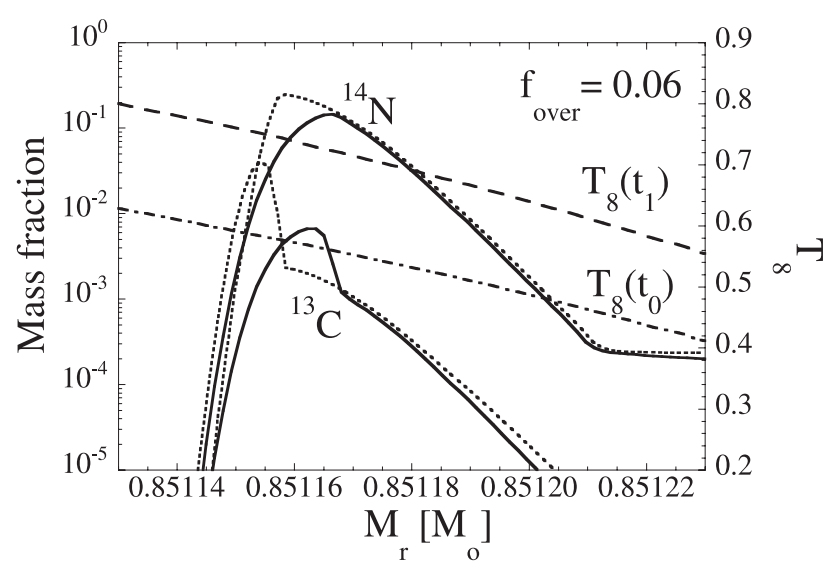

Fig. 2. Same as Fig. 1 for an overshoot with $f_{\text {over }}=0.06$.

Consequently, both the newly-formed ${ }^{13} \mathrm{C}$ and ${ }^{14} \mathrm{~N}$ also diffuse. The final ${ }^{13} \mathrm{C}$ and ${ }^{14} \mathrm{~N}$ profiles are now significantly different from the one obtained without nuclear processes accounted for during the diffusion process (dotted line in Fig. 2). In particular, the ${ }^{13} \mathrm{C}$ abundance is always lower than the ${ }^{14} \mathrm{~N}$ abundance. This effect has a drastic impact on the s-process nucleosynthesis, since wherever neutrons are produced by the ${ }^{13} \mathrm{C}(\alpha, \mathrm{n}){ }^{16} \mathrm{O}$ reaction, they will be dominantly captured by the abundant ${ }^{14} \mathrm{~N}$ rather than by the Fe seed nuclei. As shown in Fig. 3, the sprocess in this specific case is totally suppressed. The two case studies $f_{\text {over }}=0.01$ and $f_{\text {over }}=0.06$ provide extreme conditions where the diffusion process is unaffected and significantly altered by the nuclear burning, respectively. Intermediate values for the diffusive overshoot strength have also been considered and the resulting impact on the s-abundance distribution is illustrated in Fig. 3. It is clearly shown that in the specific case of this $3 M_{\odot} Z=0.0001$ stellar model in which the temperature in the overshoot region can reach values of about $T_{8}=0.4-0.8$ at the time of the 3DUP, the s-process is remarkably sensitive to the diffusive parameter $f_{\text {over }}$. Unfortunately at the present time,

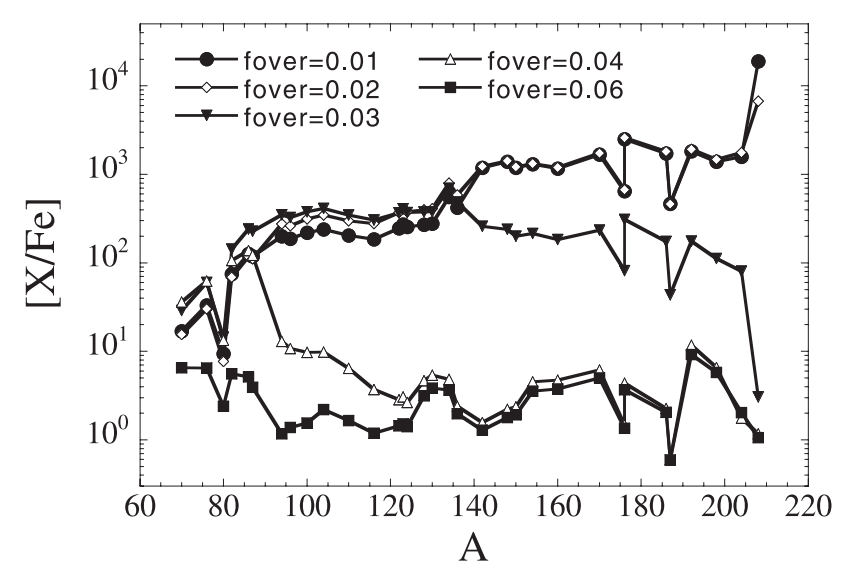

Fig. 3. Overproduction factors (with respect to solar) of s-only nuclei at the end of the interpulse phase in the partial mixing zone resulting from the diffusive overshooting model for different values of $f_{\text {over }}$. Each distribution is mass-averaged over the corresponding mixing zone $\Delta M=1,2,5,6,14 \times 10^{-5} M_{\odot}$ for $f_{\text {over }}=$ $0.01,0.02,0.03,0.04,0.06$, respectively.

no firm theoretical or observational constraints can be put on this parameter, nor on its variation with the stellar mass and metallicity.

\section{The stellar mass and stellar dependence of the s-process efficiency}

As shown in the previous section, the s-process during the radiative interpulse phase in the overshoot model is very sensitive to the temperature conditions at the time of mixing. From such calculations, it remains difficult to estimate the surface composition at the end of the AGB phase. The final s-process enrichment is further affected by the convective nucleosynthesis taking place in the pulse, and possibly by the repeated irradiations along the series of pulse-interpulse characterizing the AGB phase. The hot temperatures found at the bottom of the convective pulse are responsible for some extra production of the $A \leq 90$ isotopes and alter locally the abundance of some s-elements, but they do not modify the global s-process distribution qualitatively depicted in the previous section. As far as multiple irradiation effect is concerned, its impact strongly depends on the extent of the partial mixing zone, the overlap between successive pulses and the number of pulse-interpulse sequences. Such an effect is further affected by all the uncertainties associated with AGB modeling, including mass loss, convection and 3DUP description, as well as the unknown overshoot parameter. Despite these difficulties, we can use the discussion of Sect. 3 to sketch extreme cases: below a temperature of about $T_{8} \simeq 0.4$, diffusive overshooting is globally unaffected by nuclear burning, while above $T_{8} \simeq 0.7$, the s-process is totally inhibited. To estimate the metallicity and mass dependence of the s-process efficiency in AGB stars, we have systematically analyzed the average temperature at the base of the convective envelope at the time of the 3DUP for a grid of model stars of mass $M=1,2,3,4,5,6 M_{\odot}$ and metallicity $Z=0.02,0.008$ and 0.0001 . In this grid of models, the diffusive overshooting below the convective envelope is 


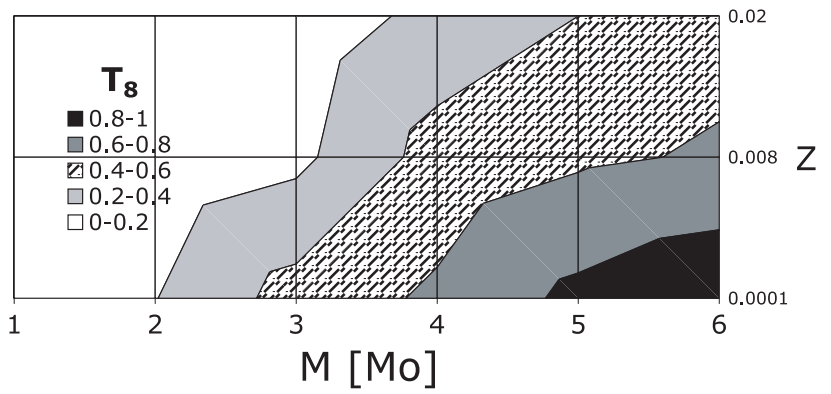

Fig. 4. Temperature at the base of the convective envelope at the time of the 3DUP for a grid of AGB models of mass $M=1,2,3,4,5,6 M_{\odot}$ and metallicity $Z=0.02,0.008$ and 0.0001 .

included since the beginning of the AGB phase with a constant value of $f_{\text {over }}=0.03$. Importantly, in none of our computations the convective envelope reaches the $\mathrm{CO}$ core, as found by Herwig (2004). The result shown in Fig. 4 provides a qualitative understanding of the mass and metallicity dependence on the s-process efficiency within the overshoot model. The hashed zone shown in Fig. $4\left(T_{8} \simeq 0.4-0.6\right)$ qualitatively delineates the transition region in the mass-metallicity plane between an efficient and suppressed s-process at a given metallicity. In particular, the s-process is not expected to be significantly affected by the conditions below the convective envelope in solar-metallicity stars less massive than $5 M_{\odot}$. On the other hand, with decreasing metallicities, the 3DUPs are hotter and the s-process efficiency decreases as well. Concerning stars in the transitional hashed zone of Fig. 4, it remains extremely difficult to predict the s-process efficiency. As shown in Sect. 3, a small modification to the unknown overshoot parameter $f_{\text {over }}$ (that could also possibly vary with the stellar mass and metallicity) can have a considerable impact on the final s-process distribution. In addition and as stressed earlier, the different nucleosynthesis calculations presented here use the same evolutionary tracks computed without overshoot. While this approach has the virtue of clearly emphasizing the strong temperature effect on the proton diffusion process, the non-negligible impact of the energy generation resulting from the proton mixing and its feedback on the stellar structure (and most particularly, the extent and duration of the 3DUP) remain to be studied in detail. This difficult study requires to couple selfconsistently the diffusion and nuclear processes within the stellar evolution code and is postponed to a future work. However, the present inconsistency does not question the previously established conclusion that the hottest the proton mixing region, the less efficient the s-process.

This picture also gives a possible explanation to some puzzling observations. In particular, the class of s-rich lowmetallicity stars that are not overabundant in $\mathrm{Pb}$ can well be understood as having been polluted by a previous generation of relatively "hot" AGB stars. As shown in Figs. 3 and 4, the s-process efficiency is expected to be relatively small in $Z=0.0001$ stars more massive than about $3 M_{\odot}$. In this model, the $\mathrm{Pb} / \mathrm{Ce}$ ratio found in the convective pulse before the 3DUP is found to be reduced from 10 to 2 for an increase of the overshoot parameter from $f_{\text {over }}=0.01$ to 0.03 . However, more quantitative determinations involving fully consistent modeling of the whole AGB phase that can explain the large surface s-enrichment observed are needed before drawing any firm conclusions about the possibility for this mechanism to explain the large range of observed $[\mathrm{Pb} / \mathrm{Ce}]$ values (e.g. Aoki et al. 2002; Van Eck et al. 2003).

\section{Conclusion}

Diffusive convective overshoot leads to the injection of protons into the adjacent ${ }^{12} \mathrm{C}$-rich core, but depending on the thermodynamic conditions in the mixing zone, the ${ }^{12} \mathrm{C}(\mathrm{p}, \gamma){ }^{13} \mathrm{~N}$ reaction can ignite during the proton diffusion. The coupling of diffusion and nuclear burning induces the overlapping of the ${ }^{14} \mathrm{~N}$-rich and ${ }^{13} \mathrm{C}$-rich layers, with drastic impact on the s-process distribution. Our study emphasizes the sensitivity of the s-process nucleosynthesis on the strength of the diffusive convective overshooting, as well as on the temperature in the corresponding region. Finally, we show that in this framework, a deep hot third dredge-up tends to inhibit the production of s-elements. We also performed a systematic analysis of the temperature at the base of the convective envelope at the time of the third dredge-up and derived qualitative estimates of the metallicity and mass dependence of the s-process efficiency in AGB stars. However, it should be stressed that the description of the s-process nucleosynthesis in AGB stars still faces many difficulties, including the nature of the mechanisms responsible for the partial mixing of protons in the C-rich layers and the uncertainties associated with mass loss, rotation, overshoot, opacities and numerics, ... that clearly need to be improved before drawing any firm quantitative conclusions.

Acknowledgements. S.G. is FNRS Research Associate. L.S. acknowledges support from the PAI V-07.

\section{References}

Aoki, W., Norris, J. E., Ryan, S. G., et al. 2002, ApJ, 580, 1149

Busso, M., Gallino, R., Lambert, D. L., Travaglio, C., \& Smith, V. V. 2001, ApJ, 557, 802

Denissenkov, P. A., \& Tout, C. A. 2003, MNRAS, 340, 722

Gallino, R., Arlandini, C., Busso, M., et al. 1998, ApJ, 497, 388

Goriely, S., \& Mowlavi, N. 2000, A\&A, 362, 599

Goriely, S., \& Siess, L. 2001, A\&A, 378, L25

Herwig, F., Blöcker, T., Schönberner, D., \& El Eid, M. 1997, A\&A, 324, L81

Herwig, F., Lugaro, M., \& Langer, N. 2003, ApJ, 593, 1056

Herwig, F. 2004, ApJ, 605, 425

Iben, I., Jr., \& Renzini, A. 1982, A\&A, 263, L23

Langer, N., Heger, A., Wellstein, S., \& Herwig, F. 1999, A\&A, 346, L37

Lugaro, M., Herwig, F., Lattanzio, J., Gallino, R., \& Straniero, O. 2003, ApJ, 586, 1305

Siess, L., Goriely, S., \& Langer, N. 2004, A\&A, 415, 1089

Van Eck, S., Goriely, S., Jorissen, A., \& Plez, B. 2001, Nature, 412, 793

Van Eck, S., Goriely, S., Jorissen, A., \& Plez, B. 2003, A\&A, 404, 291 